\title{
Divergence-free vector fields with orbital shadowing
}

Manseob Lee*

${ }^{*}$ Correspondence:

Imsds@mokwon.ac.kr

Department of Mathematics, Mokwon University, Daejeon, 302-729, Korea

\begin{abstract}
We show that a divergence-free vector field belongs to the $C^{1}$-interior of the set of divergence-free vector fields satisfying the orbital shadowing property when the vector field is Anosov.

MSC: 37C10; 37C50; 37D20

Keywords: divergence-free vector fields; star condition; shadowing; orbital shadowing; Anosov
\end{abstract}

\section{Introduction}

The shadowing theory is a very useful notion for the investigation of the stability condition. In fact, Robinson [1] and Sakai [2] proved that a diffeomorphism belongs to the $C^{1}$-interior of the set of diffeomorphisms having the shadowing property coincides the structural stability, that is, the diffeomorphism satisfies both Axiom A and the strong transversality condition. In general, if a diffeomorphism is $\Omega$-stable, that is, a diffeomorphism satisfies both Axiom A and a no-cycle condition, then there is a diffeomorphism which does not have the shadowing property (see, [3]). However, for another shadowing property, if a diffeomorphism is $\Omega$-stable, then the diffeomorphism has another shadowing property.

In this article, we study another shadowing property which is called the orbital shadowing property. It is clear that if a diffeomorphism has the shadowing property, then it has the orbital shadowing property. But the converse is not true. In fact, an irrational rotation map does not have the shadowing property, but it has the orbital shadowing property.

The orbital shadowing property was introduced by Pilyugin et al. [3]. They showed that a diffeomorphism belongs to the $C^{1}$-interior of the set of diffeomorphisms having the orbital shadowing property if and only if the diffeomorphism is structurally stable.

For a conservative diffeomorphism, Bessa [4] proved that a conservative diffeomorphism is in the $C^{1}$-interior of the set of all conservative diffeomorphisms satisfying the shadowing property if and only if it is Anosov. Lee and Lee $[5,6]$ proved that a conservative diffeomorphism is in the $C^{1}$-interior of the set of all conservative diffeomorphisms satisfying the orbital shadowing property if and only if it is Anosov. Also, for a conservative vector field, that is, a divergence-free vector field, Ferreira [7] proved that if a conservative vector field belongs to the $C^{1}$-interior of the set of all conservative vector fields satisfying the shadowing property, then it is Anosov. From the results, we study that if a conservative vector field belongs to the $C^{1}$-interior of the set of all conservative vector fields having the orbital shadowing property, then it is Anosov. Our result is a generalization of the main theorem in [7].

() 2013 Lee; licensee Springer. This is an Open Access article distributed under the terms of the Creative Commons Attribution License (http://creativecommons.org/licenses/by/2.0), which permits unrestricted use, distribution, and reproduction in any medium, provided the original work is properly cited. 


\section{Basic notions, definitions and results}

Let $M$ be a closed, connected and smooth $n(\geq 1)$-dimensional Riemannian manifold endowed with a volume form, which has a measure $\mu$, called the Lebesgue measure. Given a $C^{r}(r \geq 1)$, vector field $X: M \rightarrow T M$, the solution of the equation $x^{\prime}=X(x)$ generates a $C^{r}$ flow, $X^{t}$; on the other hand, given a $C^{r}$ flow, we can define a $C^{r-1}$ vector field by considering $X(x)=\left.\frac{d X^{t}(x)}{d t}\right|_{t=0}$. We say that $X$ is divergence-free (or a conservative vector field) if its divergence is equal to zero. Note that, by the Liouville formula, a flow $X^{t}$ is volume-preserving if and only if the corresponding vector field $X$ is divergence-free. Let $\mathfrak{X}_{\mu}^{1}(M)$ denote the space of $C^{r}$ divergence-free vector fields, and we consider the usual $C^{1}$ Whitney topology on this space. Let $X \in \mathfrak{X}_{\mu}^{1}(M)$. For any $\delta>0$ and $T>0$, we say that $\left\{\left(x_{i}, t_{i}\right): t_{i} \geq T, i \in \mathbb{Z}\right\}$ is a $(\delta, T)$-pseudo orbit of $X \in \mathfrak{X}_{\mu}^{1}(M)$ if

$$
d\left(X^{t_{i}}\left(x_{i}\right), x_{i+1}\right)<\delta
$$

for any $t_{i} \geq T, i \in \mathbb{Z}$. Define Rep as the set of increasing homeomorphisms $h: \mathbb{R} \rightarrow \mathbb{R}$ such that $h(0)=0$. Fix $\epsilon>0$ and define $\operatorname{Rep}(\epsilon)$ as follows:

$$
\operatorname{Rep}(\epsilon)=\left\{h \in \operatorname{Rep}:\left|\frac{h(t)}{t}-1\right|<\epsilon\right\} .
$$

Let $\Lambda \subseteq M$ be a compact $X^{t}$-invariant set. We say that $X^{t}$ has the shadowing property on $\Lambda$ if for any $\epsilon>0$, there is $\delta>0$ such that for any $(\delta, 1)$-pseudo orbit $\left\{\left(x_{i}, t_{i}\right)\right\}_{i \in \mathbb{Z}} \subset \Lambda$, let $T_{i}=t_{0}+t_{1}+\cdots+t_{i}$ for any $0 \leq i<b$, and $T_{i}=-t_{-1}-t_{-2}-\cdots-t_{i}$ for any $a<i \leq 0$, there exist a point $y \in M$ and an increasing homeomorphism $h: \mathbb{R} \rightarrow \mathbb{R}$ with $h(0)=0$ such that

$$
d\left(X^{h(t)}(y), X^{t-T_{i}}\left(x_{i}\right)\right)<\epsilon,
$$

for any $T_{i}<t<T_{i+1}$. If $\Lambda=M$, then $X^{t}$ has the shadowing property. Now, we introduce the notion of the orbital shadowing property. For $x \in M$, we denote $\mathcal{O}_{X}(x)$ to be the orbit of $X$ through $x$; that is, $\mathcal{O}_{X}(x)=\left\{X^{t}(x): t \in \mathbb{R}\right\}$. We say that $X^{t}$ has the orbital shadowing property if for any $\epsilon>0$ there is $\delta>0$ such that for any $(\delta, 1)$-pseudo orbit $\xi=\left\{\left(x_{i}, t_{i}\right): t_{i} \geq\right.$ $1, i \in \mathbb{Z}\}$ there is a point $y \in M$ such that

$$
\xi \subset B_{\epsilon}\left(\mathcal{O}_{X}(y)\right) \text { and } \mathcal{O}_{X}(y) \subset B_{\epsilon}(\xi)
$$

where $B_{\epsilon}(A)$ is the neighborhood of $A$. Note that the orbital shadowing property is a weak version of the shadowing property: the difference is that we do not require a point $x_{i}$ of a pseudo-orbit $\xi$ and the point $X^{t_{i}}(y)$ of an exact orbit $\mathcal{O}_{X}(y)$ to be close 'at any time moment'; instead, the sets of the points of $X$ and $\mathcal{O}_{X}(y)$ are required to be close. Let $\Lambda$ be a closed $X^{t}$-invariant set. We say that $\Lambda$ is hyperbolic if there are constants $C>0$ and $\lambda>0$ such that a continuous splitting $T_{\Lambda} M=E^{s} \oplus\langle X(x)\rangle \oplus E^{u}$ satisfying

$$
\left\|\left.D X^{t}\right|_{E^{s}(x)}\right\| \leq C e^{-\lambda t} \quad \text { and } \quad\left\|\left.D X^{-t}\right|_{E^{u}(x)}\right\| \leq C e^{-\lambda t}
$$

for any $x \in \Lambda$ and $t>0$. If $\Lambda=M$, then $X$ is called Anosov.

Given a vector field $X$, we denote by $\operatorname{Sing}(X)$ the set of singularities of $X$, i.e., those points $x \in M$ such that $X(x)=\overrightarrow{0}$. Let $R:=M \backslash \operatorname{Sing}(X)$ be the set of regular points. We know that 
the exponential map $\exp _{p}: T_{p} M(1) \rightarrow M$ is well defined for all $p \in M$, where $T_{p} M(1)=\{v \in$ $\left.T_{p} M:\|v\| \leq 1\right\}$. Given $x \in R$, we consider its normal bundle $N_{x}=\langle X(x)\rangle^{\perp} \subset T_{x} M$ and let $N_{x}(r)$ be the $r$-ball in $N_{x}$. Let $\mathcal{N}_{x, r}=\exp _{x}\left(N_{x}(r)\right)$. For any $x \in R$ and $t \in \mathbb{R}$, there are $r>0$ and a $C^{1}$ map $\tau: \mathcal{N}_{x, r} \rightarrow \mathbb{R}$ with $\tau(x)=t$ such that $X^{\tau(y)}(y) \in \mathcal{N}_{X^{t}(x), 1}$ for any $y \in \mathcal{N}_{x, r}$. We say $\tau(y)$ the first return time of $y$. Then we define the Poincarè map $f$ by

$$
\begin{aligned}
& f: \mathcal{N}_{x, r} \rightarrow \mathcal{N}_{X^{t}(x), 1}, \\
& y \mapsto f(y)=X^{\tau(y)}(y) .
\end{aligned}
$$

Let $\mathcal{N}=\bigcup_{x \in R} \mathcal{N}_{x}$ be the normal bundle based on $R$. One can define the associated linear Poincaré flow by $P_{X}^{t}(x):=\Pi_{X_{t}(x)} \circ D X^{t}(x)$, where $\Pi_{X^{t}(x)}: T_{X^{t}(x)} M \rightarrow N_{X^{t}(x)}$ is the projection along the direction of $X\left(X^{t}(x)\right)$.

Denote by int $\mathcal{O S}_{\mu}(M)$ the set of divergence-free vector fields satisfying the orbital shadowing property.

Theorem 2.1 Let $X \in \mathfrak{X}_{\mu}^{1}(M)$. If $X \in \operatorname{int} \mathcal{O S}(M)$, then $X$ has no singularity and $X$ is Anosov.

\section{Proof of Theorem 2.1}

Let $\Lambda \subset M$ be a compact, $X^{t}$-invariant and regular set. We say that $\Lambda$ is hyperbolic for $P_{X}^{t}$ if $N_{\Lambda}$ admits a $P_{X}^{t}$-invariant splitting $N_{\Lambda}=\Delta_{\Lambda}^{s} \oplus \Delta_{\Lambda}^{u}$ such that there is $l>0$ satisfying

$$
\left\|\left.P_{X}^{l}\right|_{\Delta^{s}(x)}\right\| \leq \frac{1}{2} \quad \text { and } \quad\left\|\left.P_{X}^{-l}\right|_{\Delta^{u}(x)}\right\| \leq \frac{1}{2}
$$

for all $x \in \Lambda$. The following is well known and one can find a proof in [8].

Theorem 3.1 $\Lambda$ is a hyperbolic set of $X^{t}$ if and only if the linear Poincaréflow $P_{X}^{t}$ restricted on $\Lambda$ has a hyperbolic splitting $N_{\Lambda}=\Delta^{s} \oplus \Delta^{u}$.

Consider a splitting $N=N^{1} \oplus \cdots \oplus N^{k}$ over $\Lambda$, for $1 \leq k \leq n-1$, such that all the subbundles have constant dimensions. This splitting is dominated if it is $P_{X}^{t}$-invariant, and there is $l>0$ such that for every $0 \leq i<j \leq k$, we have

$$
\left\|\left.P_{X}^{l}\right|_{N^{i}(x)}\right\| \cdot\left\|\left.P_{X}^{-l}\right|_{N^{j}\left(X^{l}(x)\right)}\right\| \leq \frac{1}{2}
$$

for any $x \in \Lambda$.

The following was proved in [9].

Theorem 3.2 [9, Proposition 4.1] If $X \in \mathfrak{X}^{1}(M)$ admits a linear hyperbolic singularity of a saddle type, then $P_{X}^{t}$ does not admit any dominated splitting over $M \backslash \operatorname{Sing}(X)$.

From the Theorem 3.2, we know that if a vector field $X$ admits a dominated splitting, then $\operatorname{Sing}(X)=\emptyset$.

Franks' lemma for divergence-free vector fields allows to realize the perturbations as perturbations of a fixed volume-preserving flow. Fix $X \in \mathfrak{X}_{\mu}^{1}(M)$ and $\tau>0$. A oneparameter area-preserving linear family $\left\{A_{t}\right\}_{t \in \mathbb{R}}$ associated to $\left\{X_{t}(p) ; t \in[0, \tau]\right\}$ is defined as follows: 
- $A_{t}: N_{p} \rightarrow N_{p}$ is a linear map for all $t \in \mathbb{R}$,

- $A_{t}=i d$, for all $t \leq 0$ and $A_{t}=A_{\tau}$ for all $t \geq \tau$,

- $A_{t} \in \mathrm{SL}(n, \mathbb{R})$ and

- the family $A_{t}$ is $C^{\infty}$ on the parameter $t$.

The following result is proved in [10, Lemma 3.2].

Lemma 3.3 Given $\epsilon>0$ and a vector field $X \in \mathfrak{X}_{\mu}^{1}(M)$, there exists $\xi_{0}=\xi_{0}(\epsilon, X)$ such that for all $\tau \in[1,2]$, for any periodic point $p$ of period greater than 2, for any sufficient small flowbox $\mathcal{T}$ of $\left\{X_{t}(p) ; t \in[0, \tau]\right\}$ and for any one-parameter linear family $\left\{A_{t}\right\}_{t \in[0, \tau]}$ such that $\left\|A_{t}^{\prime} A_{t}^{-1}\right\|<\xi_{0}$ for all $t \in[0, \tau]$, there exists $Y \in \mathfrak{X}_{\mu}^{1}(M)$ satisfying the following properties:

(a) $Y$ is $\epsilon$ - $C^{1}$-close to $X$;

(b) $Y^{t}(p)=X^{t}(p)$ for all $t \in \mathbb{R}$;

(c) $P_{Y}^{\tau}(p)=P_{X}^{\tau}(p) \circ A_{\tau}$, and

(d) $\left.\left.Y\right|_{\mathcal{T}^{c}} \equiv X\right|_{\mathcal{T}^{c}}$.

Remark 3.4 Let $X \in \mathfrak{X}_{\mu}^{1}(M)$. By Zuppa's theorem [11], we can find $Y C^{1}$-closed to $X$ such that $Y \in \mathfrak{X}_{\mu}^{\infty}(M), Y^{\pi}(p)=p$ and $P_{Y}^{\pi}(p)$ has an eigenvalue $\lambda$ with $|\lambda|=1$.

A divergence-free vector field $X$ is a divergence-free star vector field if there exists a $C^{1}$ neighborhood $\mathcal{U}(X)$ of $X$ in $\mathfrak{X}_{\mu}^{1}(M)$ such that if $Y \in \mathcal{U}(X)$, then every point in $\operatorname{Crit}(Y)$ is hyperbolic. The set of divergence-free star vector fields is denoted by $\mathcal{G}_{\mu}^{1}(M)$. Then we get the following.

Theorem 3.5 [12, Theorem 1] If $X \in \mathcal{G}_{\mu}^{1}(M)$, then $\operatorname{Sing}(X)=\emptyset$ and $X$ is Anosov.

Thus, to prove Theorem 3.7, it is enough to show that if $X$ is in the $\operatorname{int} \mathcal{O S} \mathcal{S}_{\mu}(M)$, then $X \in \mathcal{G}_{\mu}^{1}(M)$.

Lemma 3.6 If $X \in \operatorname{int} \mathcal{O S}_{\mu}^{1}(M)$, then $X \in \mathcal{G}_{\mu}^{1}(M)$.

Proof Let $X \in \operatorname{int} \mathcal{O S}_{\mu}(M)$. Then there is a $C^{1}$-neighborhood $\mathcal{U}(X)$ of $X$ such that for any $Y \in \mathcal{U}(X), Y$ has the orbital shadowing property. Let $p \in \gamma \in \operatorname{PO}\left(X_{t}\right)$ with $X^{\pi}(p)=p$ and $U_{p}$ be a small neighborhood of $p$. We will derive a contradiction. Assume that there is an eigenvalue $\lambda$ of $P_{X}^{\pi}(p)$ such that $|\lambda|=1$. By Remark 3.4, there is $Y \in \mathcal{U}(X)$ such that $Y \in \mathfrak{X}_{\mu}^{\infty}(M), Y^{\pi}(p)=p$ and $P_{Y}^{\pi}(p)$ has an eigenvalue $\lambda$ with $|\lambda|=1$. We define the map $f: \varphi_{p}^{-1}\left(N_{p}\right) \rightarrow \mathcal{N}_{p}$ with the map being the Poincarè map associated to $Y^{t}$. Here $\varphi_{p}: U_{p} \rightarrow$ $T_{p} M$ is a smooth conservative map with $\varphi_{p}(p)=\overrightarrow{0}$ (see, [13]). Let $\mathcal{V}$ be a $C^{1}$-neighborhood of $f$. Here $\mathcal{N}_{p}$ is the Poincarè section through $p$. By Lemma 3.3, we can find a small flowbox $\mathcal{T}$ of $Y^{\left[0, t_{0}\right]}, 0<t_{0}<\pi$ and there are $Z \in \mathcal{U}_{0}(Y) \subset \mathcal{U}(X), g \in \mathcal{V}$ and $\alpha>0$ such that

(a) $Z^{t}(p)=Y^{t}(p)$ for all $t \in \mathbb{R}, P_{Z}^{t_{0}}(p)=P_{Y}^{t_{0}}(p)$ and $\left.Z\right|_{\mathcal{T}^{c}}=\left.Y\right|_{\mathcal{T}^{c}}$,

(b) $g(x)=\varphi_{p}^{-1} \circ P_{Y}^{\pi}(p) \circ \varphi_{p}(x)$ for all $x \in B_{\alpha}(p) \cap \varphi_{p}^{-1}\left(N_{p}\right)$, and

(c) $g(x)=f(x)$ for all $x \notin B_{4 \alpha}(p) \cap \varphi_{p}^{-1}\left(N_{p}\right)$.

By the notion of Lemma 3.3, we can assume that $P_{Z}^{\pi}(p)$ has an eigenvalue $|\lambda|=1$. Firstly, we assume that $\lambda=1$ (the other case is similar). Then we can choose a vector $v$ associated to $\lambda$ such that $\|v\|=\alpha / 4$, and we set $\mathcal{I}_{v}=\{t \cdot v: 0 \leq t \leq 1\}$. Since $\varphi_{p}^{-1}(v) \in \varphi_{p}^{-1}\left(N_{p}\right) \backslash\{p\}$,

$$
g\left(\varphi_{p}^{-1}(v)\right)=\varphi_{p}^{-1} \circ P_{Y}^{\pi}(p) \circ \varphi_{p}\left(\varphi_{p}^{-1}(v)\right)=\varphi_{p}^{-1} \circ P_{Y}^{\pi}(p)(v)=\varphi_{p}(v) .
$$


For $0<\epsilon<\alpha / 8$, let $0<\delta<\epsilon$ be as in the definition of the orbital shadowing property of $Z^{t}$. Set $\mathcal{J}_{p}=\varphi_{p}^{-1}\left(\mathcal{I}_{v}\right)$. There is $k \in \mathbb{N}$ such that $x_{k}=\varphi_{p}^{-1}(v), v_{0}=p$ and $\left|v_{i}-v_{i+1}\right|<\delta$ for $0 \leq i \leq k-1$, where $v_{i}=t_{i} \cdot v$ for $0 \leq i \leq k-1$. We construct a $(\delta, 1)$ pseudo-orbit of $Z^{t}$ belonging to $\mathcal{J}_{p}$ as follows:

(a) $x_{i}=\varphi_{p}^{-1}\left(v_{0}\right), t_{i}=\pi$ for $i<0$,

(b) $x_{i}=g\left(\varphi_{p}^{-1}\left(v_{k}\right)\right), t_{i}=\pi$ for $0 \leq i \leq k-1$, and

(c) $x_{i}=g^{i-k}\left(\varphi_{p}^{-1}\left(v_{i}\right)\right), t_{i}=\pi$ for $i \geq k$.

Then $\xi=\left\{\left(x_{i}, t_{i}\right): i \in \mathbb{Z}\right\}$ is a $(\delta, 1)$-pseudo orbit of $Z^{t}$ and it is contained in $\mathcal{J}_{p}$. By the orbital shadowing property, we can take a point $z \in M$ such that

$$
\mathcal{O}_{Z}(z) \subset B_{\epsilon}(\xi) \text { and } \xi \subset B_{\epsilon}\left(\mathcal{O}_{Z}(z)\right)
$$

If $z \in \mathcal{J}_{p}$, then we know that there is $T_{0}>0$ such that $Z^{T_{0}}(z) \in B_{\epsilon}\left(x_{0}\right) \cap \mathcal{J}_{p}$, and $d\left(Z^{T_{0}}(z), x_{k}\right)=\alpha / 32$. Then

$$
\begin{aligned}
d\left(x_{0}, Z^{T_{0}}(z)\right) & =d\left(\varphi_{p}^{-1}\left(v_{0}\right), \varphi_{p}^{-1}\left(Z^{T_{0}}(z)\right)\right)=d\left(\varphi_{p}^{-1}(\overrightarrow{0}), \varphi_{p}^{-1}\left(Z^{T_{0}}(z)\right)\right) \\
& =d\left(p, \varphi_{p}^{-1}\left(Z^{T_{0}}(z)\right)\right)=\frac{\alpha}{8}>\epsilon .
\end{aligned}
$$

Thus $\mathcal{O}_{Z}(z) \not \subset B_{\epsilon}(\xi)$. This is a contradiction.

If $z \in M \backslash \mathcal{J}_{p}$, there is $T_{1}>0$ such that $Z^{T_{1}}(z) \in B_{\epsilon}\left(x_{0}\right)$. Then for some $j=n \pi$, we have

$$
2 \epsilon<d\left(x_{0}, x_{k}\right) \leq d\left(x_{0}, Z^{T_{1}}(z)\right)+d\left(Z^{T_{1}+j}(z), x_{k}\right)<2 \epsilon,
$$

which is a contradiction.

Finally, we assume that $\lambda$ is complex. By [10, Lemma 3.2], there is $Z \in \mathcal{U}(X)$ such that $P_{Z}^{\pi}(p)$ is a rational rotation. Then there is $l>0$ such that $P_{Z}^{l+\pi}(p)$ is the identity. Then, as in the previous argument, we get a contradiction.

End of the proof of Theorem 3.7. By Lemma 3.6, $X \in \mathcal{G}_{\mu}^{1}(M)$. Thus by Theorem 3.5, $\operatorname{Sing}(X)=\emptyset$ and $X$ is Anosov.

By [7] and our main result, we have the following.

Corollary 3.7 Let $X \in \mathfrak{X}_{\mu}^{1}(M)$. Then

$$
\operatorname{int} \mathcal{S}_{\mu}(M)=\operatorname{int} \mathcal{O S}_{\mu}^{1}(M)=\mathcal{A}_{\mu}^{1}(M),
$$

where int $\mathcal{S}_{\mu}(M)$ is the set of all divergence-free vector fields satisfying the shadowing property. 


\section{References}

1. Robinson, C: Structural stability of vector fields. Ann. Math. 99, 154-175 (1974)

2. Sakai, K: Pseudo-orbit tracing property and strong transversality of diffeomorphisms on closed manifolds. Osaka J. Math. 31, 372-386 (1994)

3. Pilygin, SY, Rodionova, AA, Sakai, K: Orbital and weak shadowing properties. Discrete Contin. Dyn. Syst. 9, 287-303 (2003)

4. Bessa, M: $C^{1}$-stable shadowable conservative diffeomorphisms are Anosov. arXiv:1112.3466

5. Lee, K, Lee, M: Symplectic diffeomorphisms with orbital shadowing. J. Chungcheong Math. Soc. 25, 739 (2012)

6. Lee, K, Lee, M: Volume-preserving diffeomorphisms with orbital shadowing. J. Inequal. Appl. 2013, 18 (2013)

7. Ferreira, C: Shadowing, expansiveness and stability of divergence-free vector fields, preprint

8. Doering, C: Persistently transitive vector fields on three-dimensional manifolds. In: Proceedings on Dynamical Systems and Bifurcation Theory, Pitmon Res. Notes Math. Ser., vol. 160, pp. 59-89 (1987)

9. Vivier, T: Projective hyperbolicity and fixed points. Ergod. Theory Dyn. Syst. 26, 923-936 (2006)

10. Bessa, M, Rocha, J: On C $C^{1}$-robust transitivity of volume-preserving flows. J. Differ. Equ. 245(11), 3127-3143 (2008)

11. Zuppa, C: Regularisation $C^{\infty}$ des champs vectoriels qui préservent lélément de volume. Bol. Soc. Bras. Mat. 10, 51-56 (1979)

12. Ferreira, C: Stability properties of divergence-free vector fields. Dyn. Syst. 27, 223-238 (2012)

13. Moser, J: On the volume elements on a manifold. Trans. Am. Math. Soc. 120, 286-294 (1965)

doi:10.1186/1687-1847-2013-132

Cite this article as: Lee: Divergence-free vector fields with orbital shadowing. Advances in Difference Equations 2013 2013:132.

\section{Submit your manuscript to a SpringerOpen ${ }^{\ominus}$ journal and benefit from:}

- Convenient online submission

- Rigorous peer review

- Immediate publication on acceptance

- Open access: articles freely available online

- High visibility within the field

- Retaining the copyright to your article 